\title{
Repulsion of an evolving surface on walls with random heights
}

\author{
L.R.G. Fontes ${ }^{\mathrm{a}, *, 1}$, M. Vachkovskaia ${ }^{\mathrm{b}, 2}$, A. Yambartsev ${ }^{\mathrm{a}, 3}$ \\ a Department of Statistics, Institute of Mathematics and Statistics, University of São Paulo, \\ rua do Matão 1010, CEP 05508-090, São Paulo SP, Brazil \\ $\mathrm{b}$ Department of Statistics, Institute of Mathematics, Statistics and Scientific Computation, University of Campinas, \\ Caixa Postal 6065, CEP 13083-970, Campinas SP, Brazil
}

Received 22 April 2004; received in revised form 18 January 2005; accepted 18 March 2005

Available online 21 June 2005

\begin{abstract}
We consider the motion of a discrete random surface interacting by exclusion with a random wall. The heights of the wall at the sites of $\mathbb{Z}^{d}$ are i.i.d. random variables. Fixed the wall configuration, the dynamics is given by the serial harness process which is not allowed to go below the wall. We study the effect of the distribution of the wall heights on the repulsion speed.

(c) 2005 Elsevier SAS. All rights reserved.
\end{abstract}

\section{Résumé}

On considère le mouvement d'une surface aléatoire discrète interagissant par exclusion avec une paroi aléatoire. Les hauteurs de la paroi sont des variables aléatoires i.i.d. Une fois fixée la configuration de la paroi, la dynamique est donnée par un processus de harnais qui ne peut descendre en dessous de la paroi. On étudie l'effet de la distribution hauteurs sur la vitesse de répulsion. (c) 2005 Elsevier SAS. All rights reserved.

MSC: 60K35; 82C41

Keywords: Harness process; Surface dynamics; Entropic repulsion; Random environment

\footnotetext{
* Corresponding author.

E-mail addresses: 1renato@ime.usp.br (L.R.G. Fontes), marinav@ime.unicamp.br (M. Vachkovskaia), yambar@ime.usp.br (A. Yambartsev).

1 Partially supported by CNPq grants 300576/92-7 and 662177/96-7 (PRONEX) and FAPESP grant 99/11962-9.

2 Partially supported FAPESP grant 99/11962-9 and CNPq grant 306029/2003-0.

3 Supported by FAPESP grant 02/01501-9.
} 


\section{Introduction}

This paper is part of a project aiming to understand the effect of the interaction with walls on the evolution of a $d$-dimensional random surface in $(d+1)$-space.

The evolving random surface is modeled by the harness process introduced by Hammersley in [9], where among other results, the fluctuations of the free case (no wall) were established in all dimensions (see also [7], where this is discussed in more detail than in here).

In [7], a solid flat wall is placed at the origin and its effect on the displacement of the surface with respect to its initial location at the origin is studied.

In that reference, it is shown that in all dimensions the average height of the surface (say, at the origin) diverges to $+\infty$ as time increases. This should be compared to the average absolute height of the surface at the origin in the free case. In the latter case, that quantity is bounded in dimensions 3 and higher [9,7]. An effect of repulsion on the wall is thus established in those dimensions. Estimates on the speed of repulsion are obtained for a class of noise distributions (including the Gaussian case). These are comparable to estimates of the entropic repulsion for the massless free field interacting with a flat wall (see [7] and references therein).

Motivated by work on the entropic repulsion for the massless free field interacting with a wall with random heights [1], we consider the same kind of wall here. In [1], estimates similar to those in [4-6] for the wall with fixed height case were obtained, showing in some cases an effect of the wall height distribution. We show the analogous effect, with analogous quantitative estimates, for the same class of noise distributions considered in [7] (see Theorem 3.1 below).

Further studies on the massless free field interacting with a wall with random heights were carried on in $[2,3]$. We refer again to [7] for other works on surfaces interacting with walls, in and out of equilibrium.

In the next section we define precisely our model and describe the flat wall result of [7], which is related and relevant to our main result. The latter is presented and argued in the following and final section. It was announced previously in [8].

\section{The model}

Denote by $|i-j|$ the number of edges in a minimal path connecting $i$ and $j$ (we will use this definition not only for $\mathbb{Z}^{d}$, but also for other graphs). Let $\mathcal{P}=\{p(i, j)\}_{i, j \in \mathbb{Z}^{d}}$ be a symmetric stochastic matrix which satisfies $p(i, j)=$ $p(0, j-i)=: p(j-i)=p(i-j)$ (homogeneity) and $p(j)=0$ for all $|j|>v$ for some $v$ (finiteness). Assume also that $\mathcal{P}$ is truly $d$-dimensional: $\left\{j \in \mathbb{Z}^{d}: p(j) \neq 0\right\}$ generates $\mathbb{Z}^{d}$. The weights $p(i, j)$ can be interpreted as transition probabilities of a random walk on $\mathbb{Z}^{d}$; denote by $\mathcal{P}$ its transition matrix and by $p_{m}(i, j)$ its $m$-step transition probabilities. By homogeneity, $p_{m}(i, j)=p_{m}(0, j-i)=: p_{m}(j-i)$.

Let $\mathcal{E}:=\left\{\varepsilon, \varepsilon_{n}(i), i \in \mathbb{Z}^{d}, n \in \mathbb{Z}\right\}$ be a family of i.i.d. integrable symmetric random variables with unbounded support. $\mathcal{E}$ represents the evolution noise variables.

We next introduce the wall variables, giving the heights at each space coordinate. Consider the family of i.i.d. random variables $\mathfrak{W}=\{W(i)\}_{i \in \mathbb{Z}^{d}}$, independent of $\mathcal{E} . W(i)$ represents the height of the wall at site $i$.

With a realization of $\mathfrak{W}$ fixed, the harness process interacting with $\mathfrak{W}$ by exclusion is defined as follows.

$$
X_{n}^{\mathfrak{W}}(i)= \begin{cases}0 \vee W(i), & \text { if } n=0 \\ W(i)+\left(\mathcal{P} X_{n-1}^{\mathfrak{W}}(i)+\varepsilon_{n}(i)-W(i)\right)^{+}, & \text {if } n \geqslant 1\end{cases}
$$

We allow $W(i)$ to take the value $-\infty$ (with positive probability), in which case the expression for $n \geqslant 1$ in (2.1) is

$$
\mathcal{P} X_{n-1}^{\mathfrak{W}}(i)+\varepsilon_{n}(i) .
$$


Remark 2.1. Notice that $X_{n}^{\mathfrak{W}}(i)$ so defined is nondecreasing in (the natural partial order for) $\mathfrak{W}$.

The case where $\mathfrak{W} \equiv-\infty$, in which we denote $X^{\mathfrak{W}}$ by $Y$, is the free case introduced by Hammersley in [9]. In that paper it is shown that $\mathbb{E} Y_{n}^{2}(0)$ is of order 1 in $d \geqslant 3$. (Notice that under our assumptions on $X_{0}^{\mathfrak{W}}$ and $\varepsilon$, $\mathbb{E} Y_{n}(0) \equiv 0$.)

The case where $\mathfrak{W} \equiv 0$, in which we denote $X^{\mathfrak{W}}$ by $Z$, was studied in [7]. We now quote some of the results of that paper, which are directly related to our main result here.

Theorem 2.1 (Part of Theorem 1.2 from [7]). Let $F_{\varepsilon}$ be the distribution function of $\varepsilon$ and define the following classes of distribution functions:

$$
\begin{aligned}
& \mathcal{L}_{\alpha}^{-}:=\left\{F: \bar{F}(x) \leqslant c \mathrm{e}^{-c^{\prime} x^{\alpha}}, x>0, \text { for some positive } c, c^{\prime}\right\}, \\
& \mathcal{L}_{\alpha}^{+}:=\left\{F: \bar{F}(x) \geqslant c \mathrm{e}^{-c^{\prime} x^{\alpha}}, x>0, \text { for some positive } c, c^{\prime}\right\},
\end{aligned}
$$

where $\bar{F}=1-F$, and

$$
\mathcal{L}_{\alpha}:=\mathcal{L}_{\alpha}^{-} \cap \mathcal{L}_{\alpha}^{+}
$$

For $d \geqslant 3$, there exist constants $c$ and $C$ that may depend on the dimension such that

(i) if $F_{\varepsilon} \in \mathcal{L}_{\alpha}$ for some $1 \leqslant \alpha \neq 1+d / 2$, then

$$
c(\log n)^{\frac{1}{\alpha}} \leqslant \mathbb{E} Z_{n}(0) \leqslant C(\log n)^{\frac{1}{\alpha} \vee \frac{2}{2+\alpha}} ;
$$

(ii) if $F_{\varepsilon} \in \mathcal{L}_{1+d / 2}$, then

$$
c(\log n)^{\frac{2}{2+d}} \leqslant \mathbb{E} Z_{n}(0) \leqslant C(\log n)^{\frac{2}{2+d}}(\log \log n)^{\frac{d}{2+d}} .
$$

\section{Results}

In the following, which is our main result, we obtain bounds on the average height of the wall at the origin as a function of $n$, the number of iterations of the dynamics, in $d \geqslant 3$. The average is taken with respect to the noise and wall variables. The bounds are similar to the corresponding ones in Theorem 2.1 above, and show an effect of the wall variables (to leading order, ignoring constants) when they have a heavy enough positive tail which is heavier than the noise ones. This is the case when the noise variables are Gaussian and the wall ones are sub-Gaussian (i.e., have distribution function belonging to $\mathcal{L}_{\theta}$ with $\theta<2$ ).

Theorem 3.1. Let $d \geqslant 3$ and suppose that $F_{\varepsilon}(x) \in \mathcal{L}_{\alpha}$ and $F_{W}(x) \in \mathcal{L}_{\theta}$. Then there exist $c$ and $C$ such that

$$
c(\log n)^{\frac{1}{\alpha} \vee \frac{1}{\theta}} \leqslant \mathbb{E} X_{n}^{\mathfrak{W}}(0) \leqslant C\left(A_{n}+(\log n)^{\frac{1}{\theta}}\right),
$$

where

$$
A_{n}= \begin{cases}(\log n)^{\frac{1}{\alpha} \vee \frac{2}{2+d}}, & \text { if } \alpha \neq 1+\frac{d}{2}, \\ (\log n)^{\frac{2}{2+d}}(\log \log n)^{\frac{d}{d+2}}, & \text { if } \alpha=1+\frac{d}{2} .\end{cases}
$$

The lower bound in (3.1) is valid for $\alpha, \theta>0$, and the upper bounds are valid for $\alpha>1, \theta>0$. 


\section{Proof.}

Lower bound. Let next $\widehat{\mathfrak{W}}=\{\widehat{W}(i)\}_{i \in \mathbb{Z}^{d}}$, where

$$
\widehat{W}(i)= \begin{cases}-\infty, & \text { if } W(i)<0, \\ 0, & \text { if } W(i) \geqslant 0 .\end{cases}
$$

It then follows that $X_{n}^{\mathfrak{W}} \geqslant X_{n}^{\widehat{\mathfrak{W}}}$. So, we need to obtain a lower bound for $\mu_{n}:=\mathbb{E} X_{n}^{\widehat{\mathfrak{W}}}(0)$.

Lemma 3.1. We have $\mu_{n} \geqslant c(\log n)^{1 / \alpha}$, where $c$ is a positive constant.

Proof. Denote $q=\mathbb{P}(W(i) \geqslant 0)$. With a slight abuse of notation we identify below $\widehat{\mathfrak{W}}$ with the set $\left\{i \in \mathbb{Z}^{d}\right.$ : $\widehat{W}(i)=0\}$. We have then

$$
\begin{aligned}
\mu_{n} & =\mathbb{E}\left(X_{n}^{\widehat{\mathfrak{W}}}(0) \mid 0 \in \widehat{\mathfrak{W}}\right) q+\mathbb{E}\left(X_{n}^{\widehat{\mathfrak{W}}}(0) \mid 0 \notin \widehat{\mathfrak{W}}\right)(1-q) \\
& =\mathbb{E}\left[\mathbb{E}\left(\left(\mathcal{P} X_{n-1}^{\widehat{\mathfrak{W}}}(0)+\varepsilon_{n}(0)\right)^{+} \mid \widehat{\mathfrak{W}}, 0 \in \widehat{\mathfrak{W}}\right)\right] q+\mathbb{E}\left[\mathbb{E}\left(\mathcal{P} X_{n-1}^{\widehat{\mathfrak{W}}}(0)+\varepsilon_{n}(0) \mid \widehat{\mathfrak{W}}, 0 \notin \widehat{\mathfrak{W}}\right)\right](1-q) \\
& =\mathbb{E} \mathcal{P} X_{n-1}^{\widehat{\mathfrak{W}}}(0)+\mathbb{E}\left[\mathbb{E}\left(\left(\mathcal{P} X_{n-1}^{\widehat{\mathfrak{W}}}(0)+\varepsilon_{n}(0)\right)^{-} \mid \widehat{\mathfrak{W}}, 0 \in \widehat{\mathfrak{W}}\right)\right] q \\
& =\mathcal{P} \mathbb{E} X_{n-1}^{\widehat{\mathfrak{W}}}(0)+\mathbb{E}\left[\mathbb{E}\left(\left(-\mathcal{P} X_{n-1}^{\widehat{\mathfrak{W}}}(0)+\varepsilon_{n}(0)\right)^{+} \mid \widehat{\mathfrak{W}}, 0 \in \widehat{\mathfrak{W}}\right)\right] q \\
& \geqslant \mathcal{P} \mathbb{E} X_{n-1}^{\widehat{\mathfrak{W}}}(0)+G\left(\mathcal{P} \mathbb{E} X_{n-1}^{\widehat{\mathfrak{W}}^{0}}(0)\right) q \\
& =\mu_{n-1}+G\left(\mathcal{P} \mathbb{E} X_{n-1}^{\widehat{\mathfrak{W}}^{0}}(0)\right) q,
\end{aligned}
$$

where $a^{-}:=a^{+}-a, G(x)=\mathbb{E}(\varepsilon-x)^{+}, \widehat{\mathfrak{W}}^{0}=\widehat{\mathfrak{W}} \cup\{0\}$, and (3.2) is due to Jensen's inequality.

We want now to estimate $\mathbb{E} X_{n}^{\widehat{\mathfrak{W}}^{0}}(j)$ in terms of $\mathbb{E} X_{n}^{\widehat{\mathfrak{W}}}(j)$. Consider the processes $X_{n}^{\widehat{\mathfrak{W}}^{0}}, X_{n}^{\widehat{\mathfrak{W}}}$ and $Y_{n}=X_{n}^{\emptyset}$ (free process), all coupled together by using the same $\varepsilon_{k}(j)$. We have that $X_{n}^{\widehat{\mathfrak{W}}^{0}}(j) \geqslant X_{n}^{\widehat{\mathfrak{W}}}(j) \geqslant Y_{n}(j)$ for all $j$. So, if $j \neq 0$, using (2.1), (2.2) and the fact that for $a \geqslant c$ it holds $(a+b)^{+}-(c+b)^{+} \leqslant a-c$, we get

$$
X_{n}^{\widehat{\mathfrak{W}}^{0}}(j)-X_{n}^{\widehat{\mathfrak{W}}}(j) \leqslant \sum_{k \in \mathbb{Z}^{d}} p(j, k)\left(X_{n-1}^{\widehat{\mathfrak{W}}^{0}}(k)-X_{n-1}^{\widehat{\mathfrak{W}}}(k)\right) .
$$

For $j=0$ we have

$$
X_{n}^{\widehat{\mathfrak{W}}^{0}}(0)-X_{n}^{\widehat{\mathfrak{W}}}(0) \leqslant \sum_{k \in \mathbb{Z}^{d}} p(0, k)\left(X_{n-1}^{\widehat{\mathfrak{W}}^{0}}(k)-X_{n-1}^{\widehat{\mathfrak{W}}}(k)\right)+\varepsilon_{n}(0)^{-}+\mathcal{P} Y_{n-1}^{-}(0)
$$

(here we used the fact that for $a \geqslant c \geqslant g$ it holds $\left.(a+b)^{+}-(c+b) \leqslant a-c+b^{-}+g^{-}\right)$. Iterating (3.4) and (3.5), one can get that

$$
X_{n}^{\widehat{\mathfrak{W}}^{0}}(j)-X_{n}^{\widehat{\mathfrak{W}}}(j) \leqslant \sum_{m=1}^{n} p_{m-1}(j)\left(\varepsilon_{n-m+1}(0)^{-}+\mathcal{P} Y_{n-m}^{-}(0)\right) .
$$

Note that, as $d \geqslant 3$, random walk with transition matrix $\mathcal{P}$ is transient and also $\mathbb{E} Y_{n}^{-}(0) \leqslant$ const, so (3.6) implies that

$$
\mathbb{E} X_{n}^{\widehat{\mathfrak{W}}^{0}}(j)-\mathbb{E} X_{n}^{\widehat{\mathfrak{W}}}(j) \leqslant C_{0}
$$

for some $C_{0}>0$, for all $n, i$, and $j$.

So, $\mu_{n}$ satisfies

$$
\mu_{n} \geqslant \mu_{n-1}+\widetilde{G}\left(\mu_{n-1}\right)
$$


where $\widetilde{G}(x)=G\left(x+C_{0}\right) q$. A lower bound of $\mathrm{O}\left((\log n)^{1 / \alpha}\right)$ for $\mathbb{E} X_{n}^{\mathfrak{W}}(0)$ then follows as in the proof of Theorem 3.1 and Corollary 3.3 in [7].

We derive next a lower bound of $\mathrm{O}\left((\log n)^{1 / \theta}\right)$. Let $\mu_{n}^{\mathfrak{W}}(i)=\mathbb{E}\left(X_{n}^{\mathfrak{W}}(i) \mid \mathfrak{W}\right)$. As the dynamics of the process can be re-written as

$$
X_{n}^{\mathfrak{W}}(i)= \begin{cases}0 \vee W(i), & \text { if } n=0, \\ \mathcal{P} X_{n-1}^{\mathfrak{W}}(i)+\varepsilon_{n}(i)+\left(W(i)-\mathcal{P} X_{n-1}^{\mathfrak{W}}(i)-\varepsilon_{n}(i)\right)^{+}, & \text {if } n \geqslant 1,\end{cases}
$$

we have

$$
\begin{aligned}
\mu_{n}^{\mathfrak{W}}(i) & =\mathcal{P} \mu_{n-1}^{\mathfrak{W}}(i)+\mathbb{E}\left(\left(W(i)-\sum_{j \in \mathbb{Z}^{d}} p(i, j) X_{n-1}^{\mathfrak{W}}(j)-\varepsilon_{n}(i)\right)^{+} \mid \mathfrak{W}\right) \\
& \geqslant \mathcal{P} \mu_{n-1}^{\mathfrak{W}}(i)+\left(W(i)-\mathcal{P} \mu_{n-1}^{\mathfrak{W}}(i)\right)^{+} .
\end{aligned}
$$

For $\mathfrak{W}$ fixed, and $i \in \mathbb{Z}^{d}$ let

$$
v_{n}^{\mathfrak{W}}(i)= \begin{cases}0 \vee W(i), & \text { if } n=0 \\ \mathcal{P} v_{n-1}^{\mathfrak{W}}(i)+\left(W(i)-\mathcal{P} v_{n-1}^{\mathfrak{W}}(i)\right)^{+}, & \text {if } n \geqslant 1\end{cases}
$$

We then have $\underset{\sim}{(\text { since }} x+(a-x)^{+}$is nondecreasing in $x$ for all $a$ ) that $\mu_{n}^{\mathfrak{W}}(i) \geqslant v_{n}^{\mathfrak{W}}(i)$ for all $\mathfrak{W}, n, i$.

Let next $\widetilde{\mathfrak{W}}=\{\widetilde{W}(i)\}_{i \in \mathbb{Z}^{d}}$, where

$$
\widetilde{W}(j)= \begin{cases}W(j), & \text { if } W(j) \geqslant 0 \\ -\infty, & \text { if } W(j)<0\end{cases}
$$

It follows that $v_{n}^{\mathfrak{W}}(i) \geqslant v_{n}^{\widetilde{\mathfrak{W}}}(i)$ for all $\mathfrak{W}, n, i$.

We will estimate $v_{n}^{\widetilde{\mathfrak{W}}}(i)$. Let us decompose $\widetilde{\mathfrak{W}}$ in the following way. $\widetilde{\mathfrak{W}}=\widetilde{\mathfrak{W}}^{i} \vee \widetilde{\mathfrak{W}}_{i}$, with $\widetilde{\mathfrak{W}}_{i}=\left\{\widetilde{W}_{i}(j)\right\}_{j \in \mathbb{Z}^{d}}$ and $\widetilde{\mathfrak{W}}^{i}=\left\{\widetilde{W}^{i}(j)\right\}_{j \in \mathbb{Z}^{d}}$, where

$$
\widetilde{W}^{i}(j)=\left\{\begin{array}{ll}
\widetilde{W}(i), & \text { if } i \neq j, \\
-\infty, & \text { if } i=j,
\end{array} \quad \widetilde{W}_{i}(j)= \begin{cases}-\infty, & \text { if } i \neq j, \\
\widetilde{W}(i), & \text { if } i=j .\end{cases}\right.
$$

Lemma 3.2. For all $n$ and $j$ it holds

$$
v_{n}^{\widetilde{\mathfrak{W}}^{i}}(j) \vee v_{n}^{\widetilde{\mathfrak{W}}_{i}}(j) \leqslant v_{n}^{\widetilde{\mathfrak{W}}}(j) \leqslant v_{n}^{\widetilde{\mathfrak{W}}^{i}}(j)+v_{n}^{\widetilde{\mathfrak{W}}_{i}}(j) .
$$

Proof. We prove the lemma by induction. For $n=0$ (3.13) is evident.

Suppose (3.13) holds for $n-1$. For $j \neq i$, we have

$$
\begin{aligned}
& v_{n}^{\widetilde{\mathfrak{W}}}(j)=\mathcal{P} v_{n-1}^{\widetilde{\mathfrak{W}}}(j)+\left(\widetilde{W}(j)-\mathcal{P} v_{n-1}^{\widetilde{\mathfrak{W}}}(j)\right)^{+}, \\
& v_{n}^{\widetilde{\mathfrak{W}}^{i}}(j)=\mathcal{P} v_{n-1}^{\widetilde{\mathfrak{W}}^{i}}(j)+\left(\widetilde{W}(j)-\mathcal{P} v_{n-1}^{\widetilde{\mathfrak{W}}^{i}}(j)\right)^{+},
\end{aligned}
$$

and

$$
v_{n}^{\widetilde{\mathfrak{W}}_{i}}(j)=\mathcal{P} v_{n-1}^{\widetilde{\mathfrak{W}}_{i}}(j) .
$$

Note that induction assumption implies $\mathcal{P} v_{n-1}^{\widetilde{\mathfrak{W}}^{i}}(j) \leqslant \mathcal{P} v_{n-1}^{\widetilde{\mathfrak{W}}_{1}}(j)$ and $\mathcal{P} v_{n-1}^{\widetilde{\mathfrak{W}}_{i}}(j) \leqslant \mathcal{P} v_{n-1}^{\widetilde{\mathfrak{W}}}(j)$. So, $v_{n}^{\widetilde{\mathfrak{W}}_{i}}(j) \leqslant v_{n}^{\widetilde{\mathfrak{W}}^{\prime}}(j)$, and, as $x+(a-x)^{+}$is increasing, $v_{n}^{\widetilde{\mathfrak{W}}^{i}}(j) \leqslant v_{n}^{\mathfrak{\mathfrak { W }}}(j)$. Also by induction assumption,

$$
\mathcal{P} v_{n-1}^{\widetilde{\mathfrak{W}}}(j) \leqslant \mathcal{P} v_{n-1}^{\widetilde{\mathfrak{W}}^{i}}(j)+\mathcal{P} v_{n-1}^{\widetilde{\mathfrak{W}}_{i}}(j)
$$


As $(a-x)^{+}$is decreasing, we have

$$
\left(\widetilde{W}(j)-\mathcal{P} v_{n-1}^{\widetilde{\mathfrak{W}}}(j)\right)^{+} \leqslant\left(\widetilde{W}(j)-\mathcal{P} v_{n-1}^{\widetilde{\mathfrak{W}}^{i}}(j)\right)^{+} .
$$

Thus,

$$
v_{n}^{\widetilde{\mathfrak{W}}}(j) \leqslant v_{n}^{\widetilde{\mathfrak{W}}^{i}}(j)+v_{n}^{\widetilde{\mathfrak{W}}_{i}}(j) .
$$

The case $j=i$ is similar.

Let us now estimate $\mathcal{P} v_{n}^{\widetilde{\mathfrak{W}}_{0}}(0)$. We suppose that $W(0)=: W>0$, otherwise $v_{n}^{\widetilde{\mathfrak{W}}_{0}} \equiv 0$. We have

$$
v_{n}^{\widetilde{\mathfrak{W}}_{0}}(i)= \begin{cases}W, & \text { if } i=0, n=0, \\ 0, & \text { if } i \neq 0, n=0, \\ \mathcal{P} v_{n-1}^{\widetilde{\mathfrak{W}}_{0}}(0)+\left(W-\mathcal{P} v_{n-1}^{\widetilde{\mathfrak{W}}_{0}}(0)\right)^{+}, & \text {if } i=0, n \geqslant 1, \\ \mathcal{P} v_{n-1}^{\widetilde{\mathfrak{W}}_{0}}(i), & \text { if } i \neq 0, n \geqslant 1 .\end{cases}
$$

It follows readily by induction that $v_{n}^{\widetilde{\mathfrak{W}}_{0}}(i) \leqslant W$ for all $i, n$. Thence, we have $v_{n}^{\widetilde{\mathfrak{W}}_{0}}(0) \equiv W$. It is now readily verified by induction that for $i \neq 0$

$$
v_{n}^{\widetilde{\mathfrak{W}}_{0}}(i)=W \sum_{k=1}^{n} p_{k}^{\{0\}}(i, 0),
$$

where, for any $j, p_{k}^{\{0\}}(j, 0)$ is the probability that the random walk with transition matrix $\mathcal{P}$ starting from $j$ returns to 0 for the first time at step $k$. It follows that

$$
\mathcal{P} v_{n}^{\widetilde{\mathfrak{W}}_{0}}(0)=W \sum_{k=1}^{n+1} p_{k}^{\{0\}}(0,0) \leqslant a W,
$$

where $a=\sum_{k=1}^{\infty} p_{k}^{\{0\}}(0,0)<1$, since $d \geqslant 3$.

By (3.13) and (3.16), we have

$$
v_{n}^{\widetilde{\mathfrak{W}}}(0) \geqslant \mathcal{P} v_{n-1}^{\widetilde{\mathfrak{W}}}(0)+\left((1-a) W-\mathcal{P} v_{n-1}^{\widetilde{\mathfrak{W}}^{\prime}}(0)\right)^{+},
$$

where $\tilde{\mathfrak{W}}^{\prime}=\left\{\widetilde{W}^{\prime}(i)\right\}_{i \in \mathbb{Z}^{d}}$, with $\widetilde{W}^{\prime}(i)=\widetilde{W}(i)$ if $i \neq 0$, and $\widetilde{W}^{\prime}(0)$ independent of $\widetilde{\mathfrak{W}}$. Taking now expectations with respect to $\tilde{\mathfrak{W}}, \widetilde{W}^{\prime}(0)$, and applying Jensen's inequality, we get

$$
\begin{aligned}
\mathbb{E} v_{n}^{\widetilde{\mathfrak{W}}}(0) & \geqslant \mathbb{E} \mathcal{P} v_{n-1}^{\widetilde{\mathfrak{W}}}(0)+\mathbb{G}\left(\mathbb{E} \mathcal{P} v_{n-1}^{\widetilde{\mathfrak{W}}{ }^{\prime}}(0)\right)=\mathcal{P} \mathbb{E} v_{n-1}^{\widetilde{\mathfrak{W}}}(0)+\mathbb{G}\left(\mathcal{P} \mathbb{E} v_{n-1}^{\widetilde{\mathfrak{W}}{ }^{\prime}}(0)\right) \\
& =\mathcal{P} \mathbb{E} v_{n-1}^{\widetilde{\mathfrak{W}}}(0)+\mathbb{G}\left(\mathcal{P} \mathbb{E} v_{n-1}^{\widetilde{\mathfrak{W}}}(0)\right)=\mathbb{E} v_{n-1}^{\widetilde{\mathfrak{W}}}(0)+\mathbb{G}\left(\mathbb{E} v_{n-1}^{\widetilde{\mathfrak{W}}}(0)\right),
\end{aligned}
$$

where $\mathbb{G}(x)=\mathbb{E}((1-a) W-x)^{+}$, and we have used the equidistribution of $\tilde{\mathfrak{W}}$ and $\tilde{\mathfrak{W}}^{\prime}$, and the translation invariance of the joint distribution of $\widetilde{\mathfrak{W}}$ and $\mathcal{E}$.

We then see that $v_{n} \equiv \mathbb{E} v_{n}^{\widetilde{\mathfrak{W}}}(0)$ is of the same form as (3.3) in [7], and a lower bound of $\mathrm{O}\left((\log n)^{1 / \theta}\right)$ for $v_{n}$ follows as in the proof of (3.4) in [7].

Upper bound. As in [7], in order to obtain an upper bound, we compare the wall process with a free process started sufficiently high. Let $X_{n}^{\mathfrak{W}, r_{n}}$ and $Y_{n}^{r_{n}}$ have the same evolution as $X_{n}^{\mathfrak{W}}$ (resp. $Y_{n}$ ), but $X_{0}^{\mathfrak{W}, r_{n}}(i)=r_{n} \vee W(i)$, $Y_{0}^{r_{n}}(i) \equiv r_{n}$. Let $\mathcal{R}_{n}=\max \{W(i):|i| \leqslant v n\}$ (recall that $v$ is a range of $\mathcal{P}$ ), and

$$
a_{n}= \begin{cases}2 K\left((\log n)^{\frac{1}{\alpha} \vee \frac{2}{2+d}}+(\log n)^{\frac{1}{\theta}}\right), & \text { if } \alpha \neq 1+\frac{d}{2}, \\ 2 K\left((\log n)^{\frac{2}{2+d}}(\log \log n)^{\frac{d}{d+2}}+(\log n)^{\frac{1}{\theta}}\right), & \text { if } \alpha=1+\frac{d}{2} .\end{cases}
$$


Note that $\mathbb{P}\left(\mathcal{R}_{n}>K(\log n)^{\frac{1}{\theta}}\right) \leqslant n^{c_{1}-c_{2} K^{\theta}}$. Take $r_{n}=a_{n} / 2$. We have

$$
\begin{aligned}
\mathbb{P}\left(X_{n}^{\mathfrak{W}}(0) \geqslant a_{n}\right) & \leqslant \mathbb{P}\left(X_{n}^{\mathfrak{W}, r_{n}}(0) \geqslant a_{n}\right) \\
& =\mathbb{P}\left(X_{n}^{\mathfrak{W}, r_{n}}(0) \geqslant a_{n}, X_{n}^{\mathfrak{W}, r_{n}}(0)=Y_{n}^{r_{n}}(0)\right)+\mathbb{P}\left(X_{n}^{\mathfrak{W}, r_{n}}(0) \geqslant a_{n}, X_{n}^{\mathfrak{W}, r_{n}}(0) \neq Y_{n}^{r_{n}}(0)\right) \\
& \leqslant \mathbb{P}\left(Y_{n}^{r_{n}}(0) \geqslant a_{n}\right)+\mathbb{P}\left(X_{n}^{\mathfrak{W}, r_{n}}(0) \neq Y_{n}^{r_{n}}(0)\right) .
\end{aligned}
$$

In Section 5 of [7] it was shown that $\mathbb{P}\left(Y_{n}^{r_{n}}(0) \geqslant a_{n}\right) \leqslant k n^{c_{3}-c_{4} K}$. As for $\mathbb{P}\left(X_{n}^{\mathfrak{W}, r_{n}}(0) \neq Y_{n}^{r_{n}}(0)\right)$, note that $X_{n}^{\mathfrak{W}, r_{n}}(0)$ and $\left.Y_{n}^{r_{n}}(0)\right)$ can be different if either if $\mathcal{R}_{n}>r_{n}$, or if it occurs

$$
\left\{Y_{l}^{r_{n}}(j)<\mathcal{R}_{n} \text { for some }(l, j) \text { with } l \leqslant n,|j| \leqslant v(n-l)\right\} .
$$

We have then

$$
\begin{aligned}
\mathbb{P}\left(Y_{l}^{r_{n}}(j)<\mathcal{R}_{n}\right) & \leqslant \mathbb{P}\left(Y_{l}^{r_{n}}(j)<K(\log n)^{\frac{1}{\theta}}\right)+\mathbb{P}\left(\mathcal{R}_{n}>K(\log n)^{\frac{1}{\theta}}\right) \\
& \leqslant \mathbb{P}\left(Y_{l}(j)<K(\log n)^{\frac{1}{\theta}}-r_{n}\right)+n^{c_{1}-c_{2} K^{\theta}} \\
& \leqslant \mathbb{P}\left(Y_{l}(j)>r_{n}-K(\log n)^{\frac{1}{\theta}}\right)+n^{c_{1}-c_{2} K^{\theta}} \\
& \leqslant k n^{c_{5}-c_{6} K^{\theta \wedge 1}}
\end{aligned}
$$

where $\theta \wedge 1=\min \{\theta, 1\}$, and

$$
\mathbb{P}\left(X_{n}^{\mathfrak{W}, r_{n}}(0) \neq Y_{n}^{r_{n}}(0)\right) \leqslant n^{c_{1}-c_{2} K^{\theta}}+\sum_{l=0}^{n} \sum_{|j| \leqslant v(n-l)} k n^{c_{5}-c_{6} K^{\theta \wedge 1}} \leqslant k^{\prime} n^{c_{7}-c_{8} K^{\theta \wedge 1}} .
$$

So,

$$
\mathbb{P}\left(X_{n}^{\mathfrak{W}}(0) \geqslant a_{n}\right) \leqslant k^{*} n^{c^{\prime}-c^{\prime \prime} K^{\theta \wedge 1}}
$$

and, by taking $K$ large enough, this implies that $\mathbb{E} X_{n}^{\mathfrak{W}}(0) \leqslant \frac{C}{2 K} a_{n}$ (see end of Section 6 of [7] for the reasoning in a similar situation).

\section{References}

[1] D. Bertacchi, G. Giacomin, Enhanced interface repulsion from quenched hard-wall randomness, Probab. Theory Relat. Fields 124 (2002) 487-516.

[2] D. Bertacchi, G. Giacomin, On the repulsion of an interface above a correlated substrate, in: Proceedings of 6th Brazilian School of Probability, Bull. Braz. Math. Soc. (N.S.) 34 (3) (2003) 401-415.

[3] D. Bertacchi, G. Giacomin, Wall repulsion and mutual interface repulsion: an harmonic crystal model in high dimensions, Stochastic Process. Appl. 110 (2004) 45-66.

[4] E. Bolthausen, J.-D. Deuschel, G. Giacomin, Entropic repulsion and the maximum of the two-dimensional harmonic crystal, Ann. Probab. 29 (4) (2001) 1670-1692.

[5] E. Bolthausen, J.-D. Deuschel, O. Zeitouni, Entropic repulsion of the lattice free field, Comm. Math. Phys. 170 (2) (1995) $417-443$.

[6] J.-D. Deuschel, Entropic repulsion of the lattice free field. II. The 0-boundary case, Comm. Math. Phys. 181 (3) (1996) $647-665$.

[7] P.A. Ferrari, L.R.G. Fontes, B. Niederhauser, M. Vachkovskaia, The serial harness interacting with a wall, Stochastic Process. Appl. 114 (2004) 175-190.

[8] L.R.G. Fontes, M. Vachkovskaia, A. Yambartsev, Entropic repulsion on a rarefied wall, in: Proceedings of the Conference "Discrete Random Walks 2003", Discrete Math. Theor. Comput. Sci. AC (2003) 105-112.

[9] J.M. Hammersley, Harnesses, in: Proc. Fifth Berkeley Sympos. Mathematical Statistics and Probability, vol. III A, 1965/66, pp. 89-117. 